\title{
Global Gradient Algorithm Extension to Distributed Pressure Driven Pipe Demand Model
}

\author{
Andrea Menapace $^{1} \cdot$ Diego Avesani $^{1}$ \\ Received: 26 March 2018 / Accepted: 17 December 2018 / \\ Published online: 19 February 2019 \\ (C) The Author(s) 2019
}

\begin{abstract}
It has been proved that the standard representation of water demand in a Water Distribution Network (WDN) leads to pipe head loss errors as well that the fully satisfied demand regardless water pressure assumption is misleading. This follows that different algorithms have been developed in order to overcome these two drawbacks although separately and independently. Consequently, this paper introduces an alternative formulation of the Global Gradient Algorithm (GGA), referred to as UD-PD, which is able to solve uniformly distributed pressure driven demands along the pipes of a WDN in extended period simulations. In addition, this new scheme is tested against reference solutions and its performances are compared with the standard WDN models. Finally, the UD-PD is applied to a real WDN under pressure deficit conditions. Numerical results show that the hydraulic heads computed with the UD-PD result higher than those simulated with standard demand driven models and that the UD-PD is able both to capture the non linear behavior of the hydraulic head along the network and to correctly compute the flow inversion even in pressure driven conditions.
\end{abstract}

Keywords Water distribution network modelling - Uniformly distributed demand · Pressure driven demand · Global Gradient Algorithm

\section{Introduction}

Nowadays, the water scarcity scenarios (Jakob and Steckel 2016) and the effects of climate change on water resources (Oki and Kanae 2006) drive the development of hydraulic tools

Electronic supplementary material The online version of this article (https://doi.org/10.1007/s11269-018-2174-3) contains supplementary material, which is available to authorized users.

Diego Avesani

diego.avesani@unibz.it

Andrea Menapace

Andrea.Menapace@natec.unibz.it

1 Faculty of Science and Technology, Universität platz 5 - piazza Università, 5, Bolzano-Bozen, 39100 Italy 
able to correctly simulate Water Distribution Networks (WDNs). It can be argued that these are mandatory steps in order to manage and prevent urban water crisis as recently recognized by the European Environmental Agency and underlined by the Water Framework Directive (Conte et al. 2012). In particular, more accurate models of pressures and flow rates in WDNs are intent on properly support water system designs, rehabilitation plans and water demands management strategies.

Since early 1930s, many network hydraulics solvers have been developed. Firstly, Cross (1936) designed a scheme based on iterative solution of flow equations in looped pipes. Afterwards, different authors applied the Newton-Raphson technique to calculate water flows and hydraulic heads in both opened and closed networks (Epp and Fowler 1970; Boulos and Altman 1991; Murty 1972). It is only with Todini and Pilati (1988)'s works that the researchers' efforts arrived to a breakthrough when the so called GlobalGradient-Algorithm (GGA) was developed. This scheme combines energy loss equations and mass balance equations providing a simultaneous solutions for nodal head and pipe flows. This method has become de-facto the standard for WDN solvers and was adopted in the widespread and open-source program EPANET (Rossman 2000). Some examples of EPANET diffusion in the scientific community as well in the WDN design and management sectors can be found in Avesani et al. (2012), where EPANET is extended in order to simulate unsteady flow in water distribution networks with variable head tanks, in Ingeduld et al. (2006), Ingeduld (2007), which uses EPANET to model both water intermittent water flows and real-time demands forecasting in WDN. Specifically, a complete and historical review about WDN solver methods can be found in Ormsbee (2006) and a survey on EPANET in Rossman (2000).

Despite the utility and feasibility of the above schemes, they presents two limits: firstly, water demands are modelled with lumped withdrawals at network nodes and not distributed along pipes. This approximation does not preserve the energy balance along pipes and leads to significant head loss errors, as reported in Berardi et al. (2010), Giustolisi and Todini (2009). Secondly, standard hydraulic network solvers are not able to represent WDNs affected by pressure deficit conditions (Giustolisi and Todini 2009).

Consequently, in the past decade, the researchers' efforts have been focused on the development of proper extensions and alternative formulations of standard WDNs models in order to overcome those two drawbacks. Starting with models able to correctly taking into account actual demand along pipes, initially Giustolisi and Todini (2009) formulated a modification of the GGA able to simulate uniformly water demand along pipes in the so called EGGA scheme. Afterward, Berardi et al. (2010) extended the EGGA to any empirical head loss monomial formula and Menapace et al. (2018) developed an EPANET extension to consider both node and uniformly distributed demand driven demands.

A second category of schemes involve instead models able to correctly consider pressure demand driven although still at the individual node level. For example, Ang and Jowitt (2006) presents a novel algorithm based on artificial reservoirs; Rossman (2007) introduces pressure dependent demands using proper emitters and Kalungi and Tanyimboh (2003)'s scheme recognizes the pressure dependency of water consumption in the solution procedure by adjusting nodal flows. Other nodal pressure driven approaches instead are based on headflow relationship embedded in hydraulic network equations. Some examples can be found in Giustolisi and Todini (2009), Tanyimboh and Templeman (2010) and Siew and Tanyimboh (2012) in which a constitutive head-flow equation allows to compute the actual withdrawn water in a node according to the nodal demand and pressure. 
Nevertheless, to the best of authors knowledge, none mathematical solution has been yet formulated capable of representing distributed water demands and pressure deficit conditions simultaneously. As a result, the aim of this paper is to developed a proper mathematical formulation in order to model distributed pressure driven demand in a WDN. The paper is organized as follows: Section 2 presents the mathematical model for the uniformly distributed pression driven head loss equation; Section 3 describes the new numerical model in order to introduce the distributed pressure driven demand into the GGA scheme; in Section 4 the new scheme is compared with reference solutions and applied to real test cases; and finally, Section 5 summaries the main finding and outcomes of the present paper.

\section{Mathematical Model}

In a real WDN, along each pipe, connections identify the exact location of water withdrawals, due for example to residential or industrial water consumptions, while the main pipes join in crossing sections which usually do not deliver water (Giustolisi et al. 2008). Usually, the first ones are called internal nodes and the seconds structural nodes. An example of representation of a pipe and its connections is reported is Fig. 1a. It has to be noticed that the actual water withdrawals depend on available hydraulic pressure at the connection point and could not fulfill the real water demands, as for example discussed in Tanyimboh and Templeman (2010) or Wu and Clark (2009).

This follows that, in a formal mathematical formulation, the function representing the water withdrawals along the generic $i j$-th pipe connecting the $i$-th and $j$-th nodes has to be written as:

$$
w_{i j}(h(x))=\sum_{k=1}^{n_{w, i j}} \int_{-\infty}^{\infty} w_{k}\left(h\left(x_{k}\right)\right) \delta\left(x-x_{k}\right) ;
$$

where $x$ is the pipe linear coordinate whose origin is in the node $i ; w_{k}$ is the $k$-th withdrawal at the location $x_{k} ; h_{k}$ is the local hydraulic pressure in $x_{k} ; n_{w, i j}$ is the number of withdrawals in the $i j$-th pipe; and $\delta$ is the Dirac delta function, whose definition can be found in Dirac (1939). Thus, according to the Eq. 1, the total amount of water withdrawn in the $i j$-th pipe assumes the following expression:

$$
\begin{aligned}
W_{i j, t o t} & =\int_{0}^{L_{i j}} w_{i j}(h(x)) d x ; \\
& =\sum_{k=1}^{n_{w_{i j}}} w_{k}\left(h\left(x_{k}\right)\right) ;
\end{aligned}
$$

and the pipe discharge at the distance $x$ from the initial node should be written as:

$$
Q_{i j}(x)=Q_{i j}-\int_{0}^{x} w_{i j}(h(\xi)) d \xi ;
$$

where $\xi$ is spatial coordinate, $Q_{i j}$ is the flow of $i j$-th pipe in the node $i$-th. This follows that the total amount of withdrawn water in the pipe $i j-t h, W_{i j, t o t}$ equals the total amount of demand water in the corresponding pipe, i.e. $D_{i j, t o t}=W_{i j, t o t}$ only when there is sufficient pressure in each withdrawal points.

It has to be noticed that from a mathematical point of view both Eqs. 1 and 3 are discontinuous functions. Specifically, Eq. 3 is a step function of general validity, which is able to properly taken into account the flow direction along each pipes even when a flow inversion occurs and Eq. 1 is a sampling function. It is obvious that this topological representation, which is provided by Eqs. 1 and 3 and shown in Fig. 1, is correct and accurate although it 


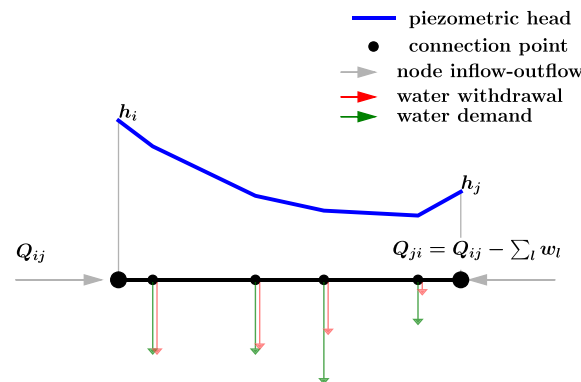

(a)

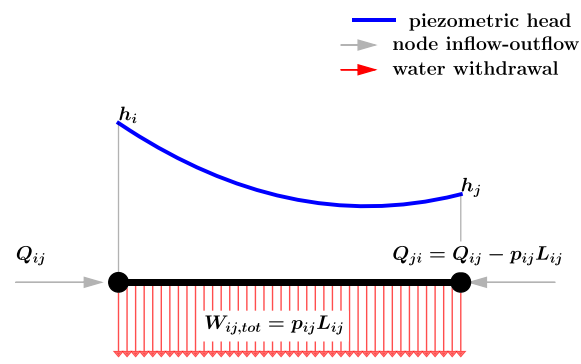

(c)

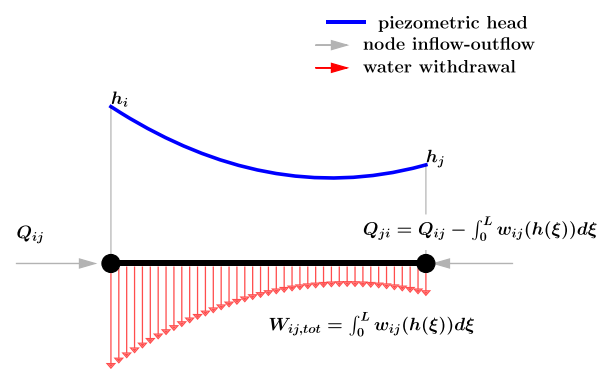

(d)

Fig. 1 Schematisation of: a Actual pipe connections, water demands and water withdrawals; b standard water withdrawas; c uniformaly demand driven water withdrawals; d uniformaly pression driven water withdrawals

presents two drawbacks. First, the discontinuity in the flow and in the withdrawal force to split the problem in a number of joint pipe equal to the number of connections. This increase the complexity in the topological representation of the WDN reducing the computational efficiency. Second, in real applications and network analysis there is the assumption of uniformly distributed water demands along the pipe due to the lack of information about the actual connections and demands.

In order to overcome this problem and at the same time to preserve the network topology, standard schemes, such as the GGA, adopt some important simplifications; the water withdrawals are consider pressure independent, namely equal to water demands (i.e. $\left.w_{i j, k}(h(x))=d_{i j, k}\right)$, where $d_{i j, k}$ is $k$-th point water demand in the $i j$-th pipe; and the total water demand $W_{i j, t o t}$ is equally split $\left(W_{i j, t o t} / 2\right)$ between the nodes $i$ and $j$. This preserve the mass balance and reduces the flow in each pipe to a continuous and constant function, which reads as:

$$
Q_{i j}(x)=Q_{i j}=\bar{Q}_{i j} .
$$

Thus, the head loss slope due to friction along a pipe in steady flow conditions read as

$$
J(x)=-\frac{d}{d x} h(x)=-r_{i j}(x)\left|\bar{Q}_{i j}\right|^{n-1} \bar{Q}_{i j}
$$


where $n \in[1,2]$ depends on the chosen mathematical expression and $r_{i j}$ is the. For example in the standard Darcy-Weisbach formula $n$ is set equal to 2, while in the Hazen-Williams expression to 1.852 (Wu and Clark 2009). Furthermore, the term $r_{i j}(x)$ is the resistance coefficient which depends on the flow regime, on the pipe equivalent roughness, and on the pipe diameter. However, in common practice, empirical formulations are adopted where the unitary hydraulic resistance is assumed constant, i.e. $r_{i j}(x)=r_{i j}$, along each pipes and independent from the flow regime. Thus the total head loss in the $i j-t h$ pipes is:

$$
h_{i}-h_{j}=\int_{0}^{L_{i j}} r_{i j} \bar{Q}_{i j}\left|\bar{Q}_{i j}\right| d x ;
$$

which reduces to:

$$
h_{i}-h_{j}=R_{i j} \bar{Q}_{i j}\left|\bar{Q}_{i j}\right|^{n-1}
$$

where $R_{i j}=L_{i j} r_{i j}$.

On the other hand, following Giustolisi (2010), in case of pressure independent demand uniformly distributed along the $i j$-th pipe it is easy to prove that:

$$
Q_{i j}(x)=Q_{i j}-q_{i j} x ;
$$

where $q_{i j}$ is the non negative demand per unit length in the $i j-t h$ pipe and is equal to

$$
q_{i j}=\frac{W_{i j, t o t}}{L_{i j}} .
$$

As in the node demand model, Eq. 8 results continuous, which implies that can be directly integrated, and varies linearly along the pipe. This follows that the total head loss can be directly computed as:

$$
\begin{aligned}
h_{i}-h_{j} & =\int_{0}^{L_{i j}} r_{i j}\left(Q_{i j}-q_{i j} x\right)\left|\left(Q_{i j}-q_{i j} x\right)\right|^{n-1} d x ; \\
& =\frac{r_{i j}}{q_{i j}} \frac{\left|Q_{i j}\right|^{n+1}-\left|Q_{j i}\right|^{n+1}}{n+1} ;
\end{aligned}
$$

where $Q_{j i}$ is the flow of $i j$-th pipe in the $j$-th node. As reported in Giustolisi and Todini (2009), Giustolisi (2010), the Expression (10) preserves both mass and energy.

Nevertheless, in case of uniformly distributed pressure driven demand, the actual water withdrawal is not constant, as in the uniformly distributed demand driven case, although it is still a continuous function. As a result, the head loss in the $i j-t h$ pipe has to be rewritten as presented in Eq. 11 in order to properly taking into account the water withdrawn dependency on hydraulic pressure:

$$
h_{i}-h_{j}=\int_{0}^{L_{i j}} r(x)\left(Q_{i j}-\int_{0}^{x} w_{i j}(h(\xi)) d \xi\right)\left|Q_{i j}-\int_{0}^{x} w_{i j}(h(\xi) d \xi)\right|^{n-1} d x .
$$

It is worth noting that the Eq. 11 is a function whose solution depends on the function itself and consequently admits a direct solution only in very simple cases: firstly, when the withdrawal is null, which reduces (11) to nodal demand case; secondly when withdrawal is constant, namely constant water demand, which leads to the Eq. 10. For sake of completeness, Eq. 11 admits still a direct solutions when it assumes the Volterra integral formulation (Press et al. 2007) which is nevertheless not applicable to the WDN equations.

Nevertheless, differently from the original (1), the $w_{i j}$ function results now continuous and differentiable. In particular it could be approximated within an iterative scheme like those characterizing WDN hydraulic solvers. Therefore, the novel idea presented in this paper is to developed a strategy in order to compute an approximate $w_{i j}$ function which still 
allows a correct simulation of the head loss in a pipe. In particular, the core of the scheme is borrowed from high order numerical Finite Element Methods (FEM) where high order polynomials approximate the exact solution in each mesh element from the values computed in the nodes of the mesh by the FEM solver.

\section{The New Scheme for Distributed Pressure Driven Demand}

In FEMs, the computational domain is divided in elements with nodes. According to the approximation order, each element has additional nodes where the numerical solution has to be computes. For example, Fig. 2a shows a two-dimensional domain divided in four elements, where each element has four additional nodes which allow to compute second order polynomials in order to approximate the analytical solution of the problem. An example of second order approximating function is reported in Fig. 2c.

As in the FEM-based schemes, WDN are made by nodes linked not with multidimensional elements but with one dimensional element, i.e. the pipes, where an arbitrary number

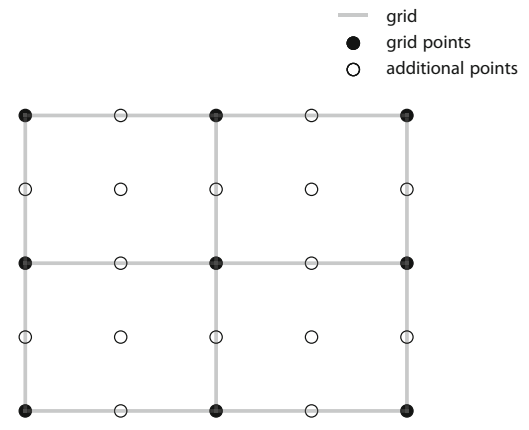

(a) Example of two dimesional finite element grid.

(c) Example of one dimesional basis functions.

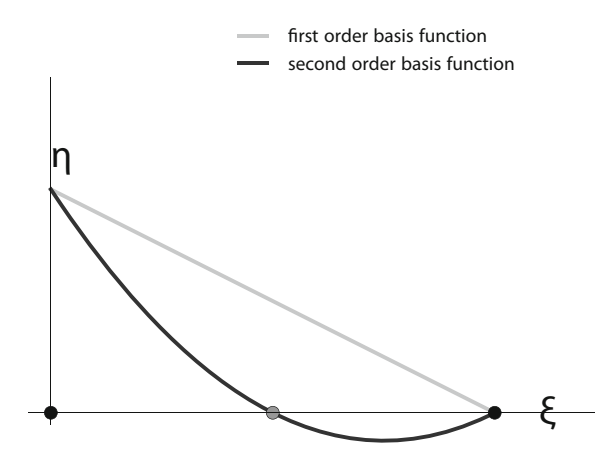

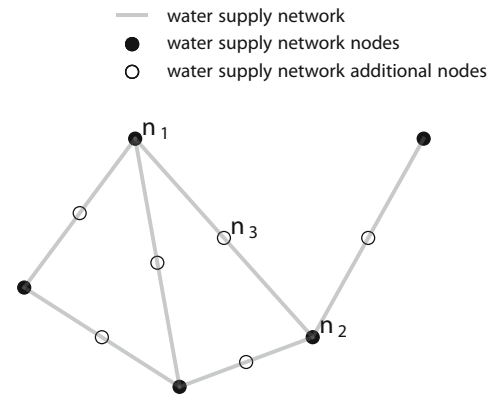

(b) Example of water supply system network.
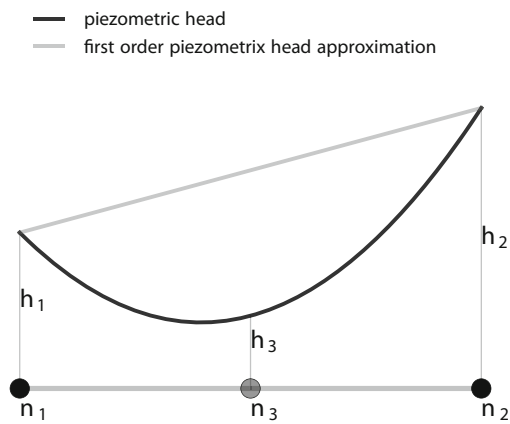

(d) Example of piezometric head distribution.

Fig. 2 A comparison between finite element nodes displacment and water supply system junctions 
of additional nodes can be added in order to reconstruct the water withdrawal function, known the pressure head in each nodes, as shown in Fig. 2b and d. It follows that, starting from the classical Global Gradient Algorithm, each iterative step could provide the solution of the hydraulic head both in water supply network nodes and in the pipes additional nodes. After that, known the hydraulic head and chosen a proper head-flow relationship such as those described in nodal pressure demand schemes, the withdrawal function along each pipe of the network can be approximated.

Specifically, following the general FEM formulation, the actual water demand function $w_{i j}(x)$ along the $i j$-th pipe is approximated with a generic polynomial function $\widetilde{w}_{i j}(x)$ which reads as:

$$
\widetilde{w}_{i j}(x)=\sum_{l=1}^{n_{d}} \widetilde{w}_{i j, l} x^{l-1}
$$

where the terms $\widetilde{w}_{i j, l}$ denotes the set of unknown coefficients of the reconstruction polynomial for each $i j$-th pipe and $n_{d}$ are the total number of point which have to be consider in each pipes according to the polynomial order $M$. Namely, $n_{d}$ is equal to $M+1$. It is clear that $n_{d}$ include both the pipe initial node $i$ and final node $j$. For sake of clarification, Appendix A describes the general procedure to compute the unknown coefficient of the reconstructed withdrawal function $\widetilde{w}_{i j}(x)$ of arbitrary order of approximation and more in details illustrates the case of second order appropriation.

Hence, according to the approximation given by the Eq. 12, the Eq. 11 can be re-written as:

$$
h_{i}-h_{j}=\int_{0}^{L_{i j}} r_{i j}\left(Q_{i j}-\int_{0}^{x} \widetilde{w}_{i j}(\xi) d \xi\right)\left|Q_{i j}-\int_{0}^{x} \widetilde{w}_{i j}(\xi) d \xi\right|^{n-1} d x
$$

which reduces to:

$h_{i}-h_{j}=\int_{0}^{L_{i j}} r_{i j}\left(Q_{i j}-\sum_{l=1}^{M+1} \frac{1}{1+(l-1)} \widetilde{w}_{i j, l} x^{l}\right)\left|Q_{i j}-\sum_{l=1}^{M+1} \frac{1}{1+(l-1)} \widetilde{w}_{i j, l} x^{l}\right|^{n-1} d x$.

It is important to underline that Eq. 14 is directly integrable, different against Eq. 11, and can be used to compute the head loss along the $i j$-th pipe connecting the $i$-th and $j$-th node, known the flow $Q_{i j}$ in the node $i$-th and the withdrawal along the pipe.

As a matter of fact, the head loss in the $i j-t h$ pipe is a known function $\mathcal{H}$ which can be written as:

$$
h_{i}-h_{j}=\mathcal{H}\left(R_{i j}, L_{i j}, \widetilde{w}_{i j, l}, Q_{i j}\right)
$$

where the total piezometric head-loss, i.e. $\Delta h_{i j}=h_{i}-h_{j}$, depends on the resistance closure formula, on the length of the $i j$-th pipe and on the coefficients of the approximated water demand function which has been computed at the previous iterative step. It worth noting that the function $\mathcal{H}$ results continuous and differentiable and known once choosen the appropriation order for the withdrawal function.

Consequently, it is possible to write the flow problem in a generic WDN as:

$$
\begin{cases}\Delta h_{i j}=\mathcal{H}\left(R_{i j}, L_{i j}, \widetilde{w}_{i j, l}, Q_{i j}\right) ; & \forall i \cap j \neq \emptyset \\ \sum_{i}\left(Q_{i \hat{\imath}}-W_{i \hat{\imath}}\right)-\sum_{j} Q_{\hat{\imath} j}=0 ; & \forall i \cap \hat{\imath} \vee \hat{\imath} \cap j \neq \emptyset \\ h_{i j, l}=h_{i}-\mathcal{H}\left(R_{i j}, x_{k}, \widetilde{w}_{i j, l}, Q_{i j}\right) ; & \forall i \cap j \neq \emptyset \wedge l \in\left[1, n_{i j, l}\right] \\ & i, j \in\left[1, n_{t}\right] ; \hat{\imath} \in\left[1, n_{n}\right] ;\end{cases}
$$


where $n_{i j, l}$ is the number of withdrawal polynomial points, $n_{t}$ is the total number of nodes and $n_{n}$ is the total number of junction, i.e., the number of nodes where the piezometric head is unknown. According to the standard formulation, Eq. 16a describes the head losses along each pipe of the network and Eq. 16b represents the mass balance at each of the unknown head nodes. On the other hand, differently from the origial formulation, the Eq. 16c has been also introduced in order to properly compute the unknown pressure dependent withdrawal function along each pipe. This follows that the System (16) is a non linear system of equations which, as for the standard GGA scheme, can be linearized using the Taylor series expansion.

Hence, the head loss equation at iteration step $f+1$ is therefore written as:

$$
\begin{gathered}
\Delta h_{i j}\left|Q_{i}^{f+1}=\Delta h_{i j}\right|_{Q_{i}^{f}}+\left.\left(Q_{i j}^{f+1}-Q_{i j}^{f}\right) \frac{d}{d Q_{i j}} \Delta h_{i j}\right|_{Q_{i}^{f}} ; \\
Q_{i j}^{f+1}=\Delta h_{i j}\left|{ }_{Q_{i j}^{f+1}}\left(\left.\frac{d}{d Q_{i j}} \Delta h_{i j}\right|_{Q_{i j}^{f}}\right)^{-1}-\Delta h_{i j}\right|_{Q_{i j}^{f}}\left(\left.\frac{d}{d Q_{i j}} \Delta h_{i j}\right|_{Q_{i j}^{f}}\right)^{-1}+Q_{i j}^{f} ;
\end{gathered}
$$

Simplifying the notation, the Eq. 18 is written as:

$$
Q_{i j}^{f+1}=\left(h_{i}^{f+1}-h_{j}^{f+1}\right) a_{i j}^{f}-b_{i j}^{f} ;
$$

where

$$
a_{i j}^{f}=\left(\frac{d}{d Q_{i j}} \Delta h_{i j} \mid{ }_{Q_{i j}^{f}}\right)^{-1} ;
$$

and

$$
b_{i j}^{f}=a_{i j}^{f}\left(h_{i}^{f}-h_{j}^{f}\right) .
$$

Therefore the final iterative schemes results:

$$
\begin{cases}h_{l, i j}^{f}=h_{i}-\mathcal{H}\left(R_{k}, x_{i j, l}, \widetilde{w}_{i j, l}^{f}, Q_{i j}^{f}\right) ; & \forall i \cap j \neq \emptyset \wedge l \in\left[1, n_{i j, p}\right] \\ Q_{i j}^{f+1}=\left(h_{i}^{f+1}-h_{j}^{f+1}\right) a_{i j}^{f}+b_{i j}^{f} ; & \forall i \cap j \neq \emptyset \\ \sum_{i} Q_{i \hat{\imath}}^{f+1}-\sum_{j} Q_{\hat{\imath} j}^{f+1}=0 ; & \forall i \cap \hat{\imath} \vee \hat{\imath} \cap j \neq \emptyset ; \\ & i, j \in\left[1, n_{t}\right] ; \hat{\imath} \in\left[1, n_{n}\right] ;\end{cases}
$$

which can be directly cast inside the GGA procedure where only one additional step in the iterative procedure has to be considered. In order to facilitate the developed and the explanation of the new scheme, we limit ourself to a general discussion of the method. However, Appendix B reports the Equations in case of second order approximation for water withdrawals, flow rate and head loss in a generic pipe. The coefficients for Taylor series expansion are also derived.

\section{Test Cases}

In the following Section the new distributed pressure driven scheme is tested with reference solutions and its effectiveness is compared with standard nodal demand scheme, nodal pressure driven scheme and distributed demand driven scheme. Without loss of generality, in all 
the following test cases the Darcy-Weisbach equation has been used to model the head loss and the following pressure demand-relationship is considered:

$$
w_{k}=q_{k} \frac{\exp \left(\alpha+\beta p_{k}\right)}{1+\exp \left(\alpha+\beta p_{k}\right)}
$$

where $p_{k}=h_{k}-z_{k}$ with $z_{k}$ the local geodetic elevation. Moreover, $\alpha$ and $\beta$ are defined respectively as (Tanyimboh and Templeman 2010; Siew and Tanyimboh 2012):

$$
\begin{gathered}
\alpha=\frac{-4.595 p^{r}-6.907 p^{\min }}{p^{r}-p^{\min }} ; \\
\beta=\frac{11.502}{p^{r}-p^{\min }} .
\end{gathered}
$$

In Eqs. 24 and 25, the term $p^{\text {min }}$ is the hydraulic pressure below which outflow is zero and $p^{r}$ is the pressure for full demand satisfaction. In addition, a second order of approximation has been used in order to approximate the withdrawal function. For the sake of clarity, we call hereafter the nodal demand driven scheme N-DD; the nodal pressure driven scheme NPD; the uniformly distributed demand driven scheme UD-DD; and the uniformly distributed pressure driven scheme UD-PD.

\subsection{Reference Test Case}

The aim of this test case is to verified the present new UD-PD scheme with a reference solution. In particular, the test consists in simulating the pressure and the flow rate in a single dead-end pipe connecting two nodes, named $i$ and $j$, where the hydraulic pressure in the node $i$ and the water demand are the only known variables of the problem. In order to compare the solution obtained with the different methods the following reference solution is considered: the $i j$ pipe is divided with 100 nodes equally distributed which have a water demand of $0.1 \mathrm{l} / \mathrm{s}$. This follows that it is possible to solve the standard head loss equation, where the flow rate is constant, for each section of the pipe and to apply the mass balance in each nodes sequentially starting from the initial node $i$ where the pressure is known. In particular, it is possible to calculate in each node the actual withdrawal, which depends locally on the pressure as described by the Eq. 23, and through an appropriate mass balance, know the water passing through the next node.

Figure $3 \mathrm{a}$ and $\mathrm{b}$ show the comparison between the reference solution, the N-DD, N-PD, UD-DD and UD-PD models for both the hydraulic heads and water flow along the pipe. It can be observed that the new UD-PD scheme reproduces accurately the reference solutions for both hydraulic pressure and water flow. On the contrary, the other schemes are not able to represent the solution. As expected, the hydraulic heads computed with both the nodal demand base models (i.e. N-DD, N-PD schemes) are linear and are not able to capture the non linear behaviour of the solution. On the other hand, despite the hydraulic head computed with the UD-DD model is cubic, according to Eq. 10, it still underestimates the reference solutions. The same drawbacks can be found also in the water flow solution: the nodal base model consider a constant flow along the pipe while the UD-DD reproduces a water flow that varies linearly along the pipe. Also in this case, the UD-PD model is the only model that correctly reproduces the reference solutions. 


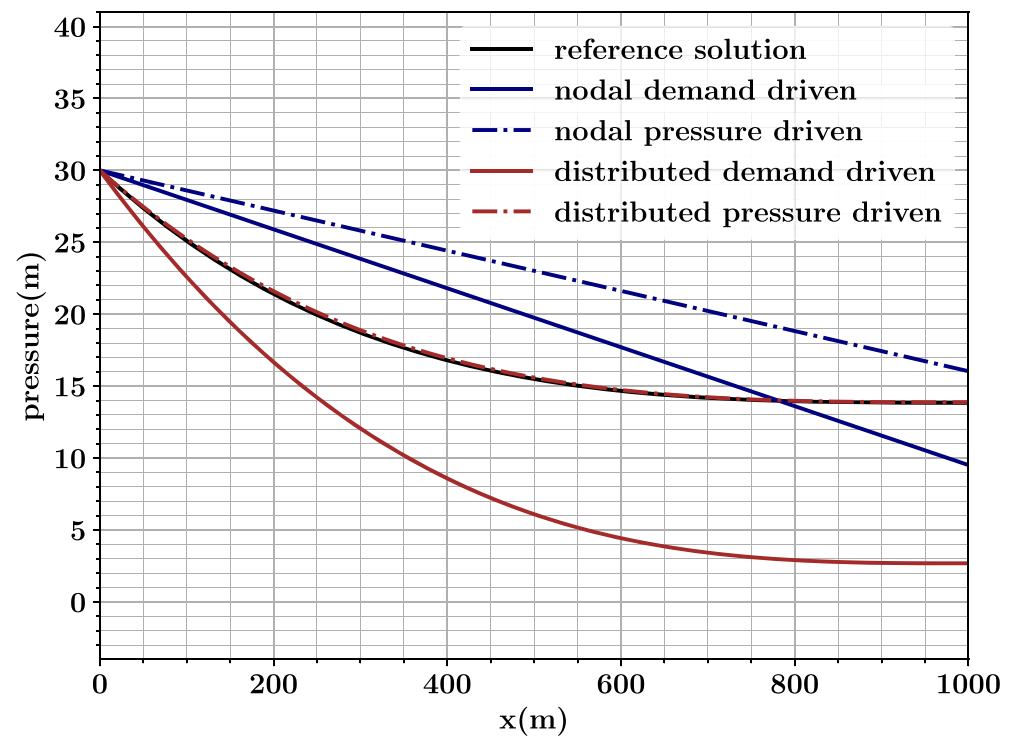

(a)

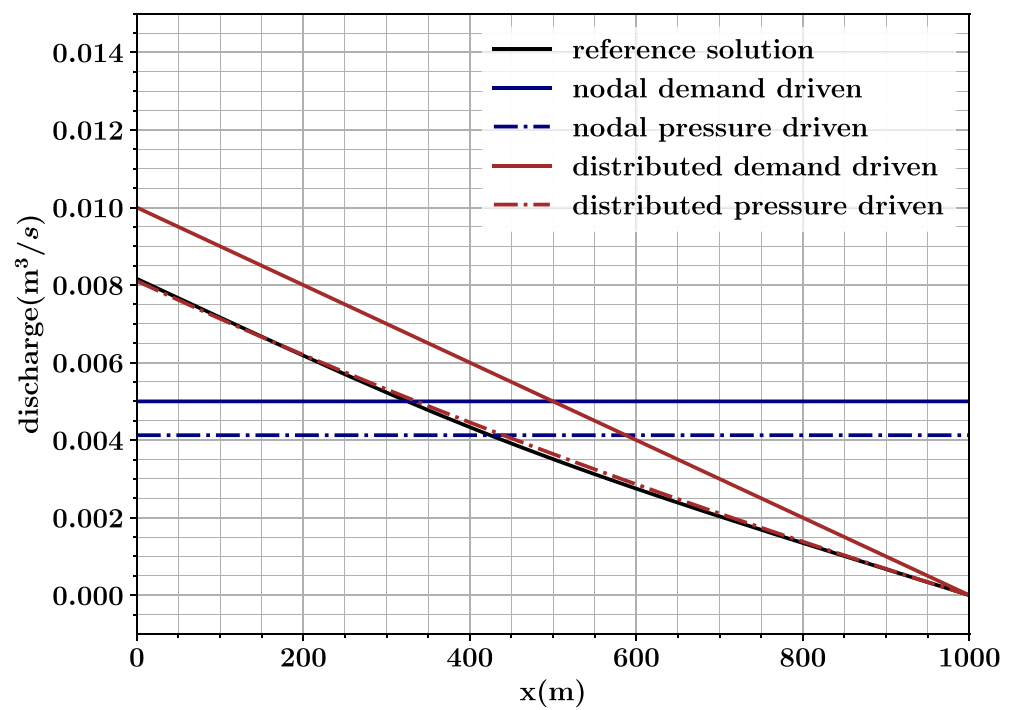

(b)

Fig. 3 Comparison between different scheme for pressure (a) and flow (b) in a reference test case

\subsection{Two Tank Test Case}

In order to discuss the implications of the new UD-PD scheme, a simplify WDN is considered. It consists in two tanks, named $T 01$ and $T 02$, connected with three pipes (see layout 
and detailed report of the network in Fig. 8 and Table 1 of Electronic Supplementary Material). In order to cover both demand driven and pressure deficit conditions, four scenarios are simulated. In the first case, case $(i)$, the water levels is set at $80 \mathrm{~m}$ for the tank $T 01$ and $70 \mathrm{~m}$ for the tank $T 02$ while in the second case, case(ii), the water levels are set at $30 \mathrm{~m}$ and $20 \mathrm{~m}$ respectively for the tank $T 01$ and the tank $T 02$ (see Table 1 in Supplementary Material). In both cases two water total demands are taken into account: $D_{t o t, A B}=5 \mathrm{l} / \mathrm{s}$ and $D_{t o t, A B}=20 \mathrm{l} / \mathrm{s}$. These configurations have been specifically chosen because they allow to compare the presented UD-PD scheme, where the total water demand is uniformly distributed along the pipe $A B$ and is pressure driven, with both the standard uniformly distributed and demand driven schemes. In the N-DD, the total demand equally distributed in both node $A$ and $B$.

Figure 4 illustrates the hydraulic piezometric head along the network pipes and the node $A$ and $B$ computed with the N-DD, UD-DD and UD-PD in the four scenarios. According to withdrawal-head relationship in case $(i)$ tank $T 01$ and tank $T 02$ are able to deliver all the water demand and there are no pressure deficit conditions. Regarding the first configuration, Fig. 4a shows that: the tank $T 01$ supplies all the demand water; there is no flow

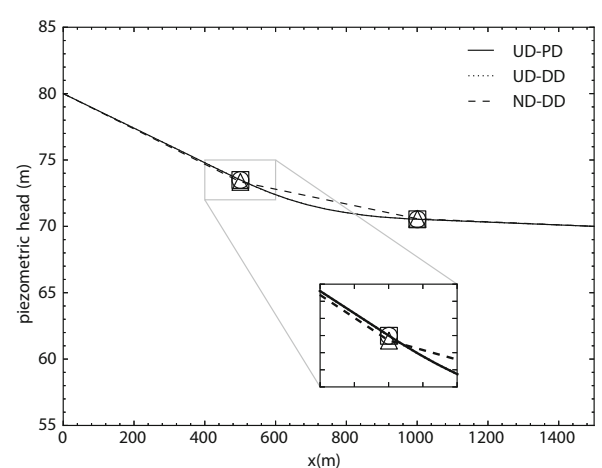

(a)

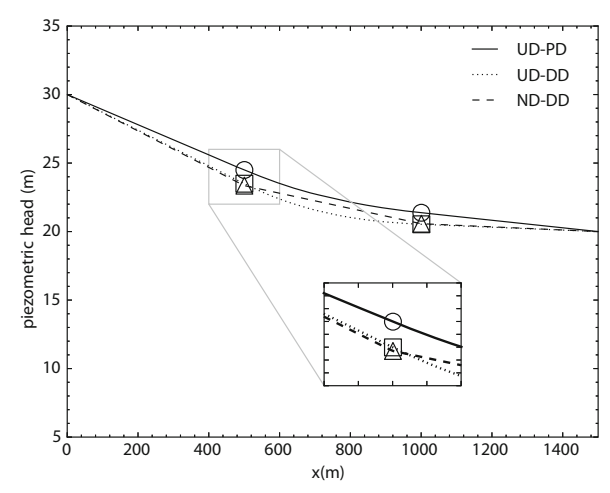

(c)

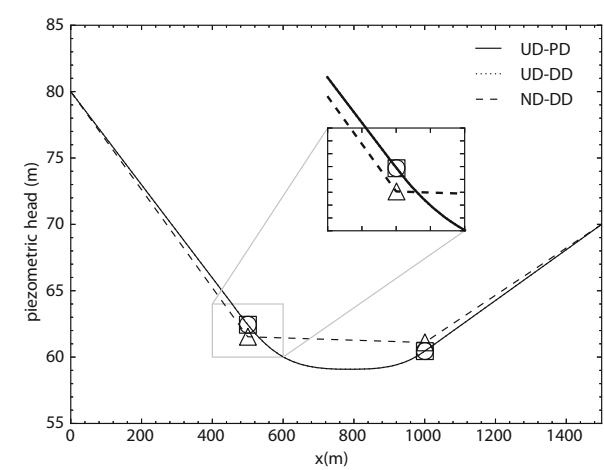

(b)

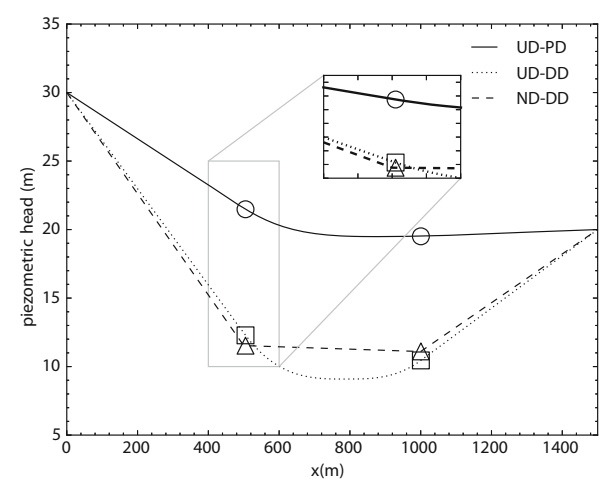

(d)

Fig. 4 Case (i) configurations: a $D_{t o t, A B}=5 \mathrm{l} / \mathrm{s}$ and $\mathbf{b} D_{t o t, A B}=20 \mathrm{l} / \mathrm{s}$. Case (ii) configurations: c $D_{\text {tot }, A B}=5 \mathrm{l} / \mathrm{s}$ and $\mathbf{d} D_{t o t, A B}=20 \mathrm{l} / \mathrm{s}$ 
inversion; and all the model are able to compute the same pressure in both nodes $A$ and $B$. As expected, the pressure along the pipe $A B$ computed with the N-DD scheme differs from the pressure derived with the UD-DD and UD-PD schemes. This differences has been already discussed in Giustolisi et al. (2008), Giustolisi and Todini (2009) and consequently no further details are here necessary. On the contrary, Fig. 4b shows negative grade of the hydraulic pressure in the pipe $B-T 02$ computed with the UD-DD and UD-PD models when the water demand increases to $D_{t o t, A B}=20$. This means that both tank $T 01$ and $T 02$ cover the water requested in pipe $A B$ resulting in a flow inversion in the pipe $A B$. This produce a small difference between the pressure computed with the three different model which amplify along the pipe. It is important to noticed that the hydraulic head computed with the UD-PD scheme corresponds to the one computed with the UD-DD scheme in both water demand scenarios proving that the new UD-PD scheme represents a generalization of the UD-DD model.

Moving to case (ii), the pressure drops and the two tanks do not ensure enough pressure to satisfy the water demand. This implies that the differences between the N-DD, UD-DD and UD-PD models increase, as reported in Fig. $4 \mathrm{c}$ and d. Focusing on $D_{t o t, A B}=5 \mathrm{l} / \mathrm{s}$ demand,

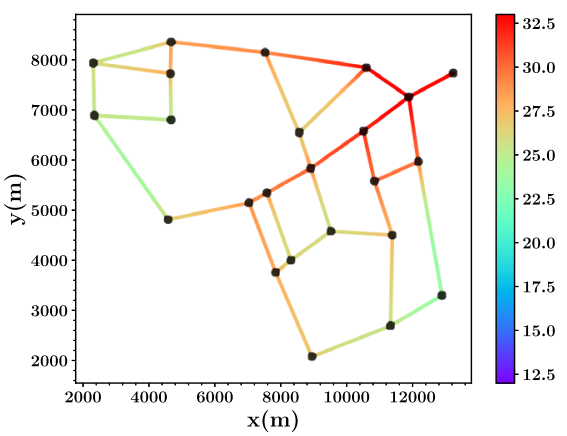

(a)

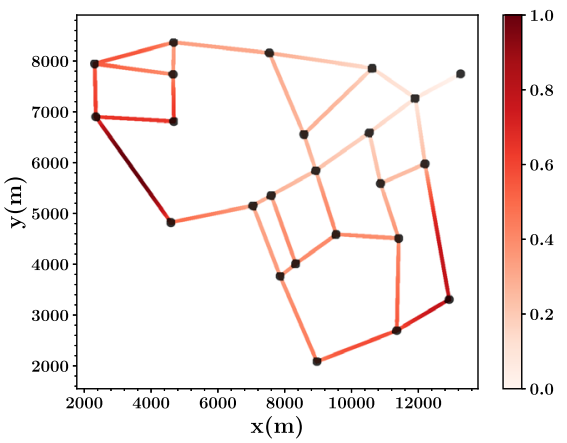

(c)

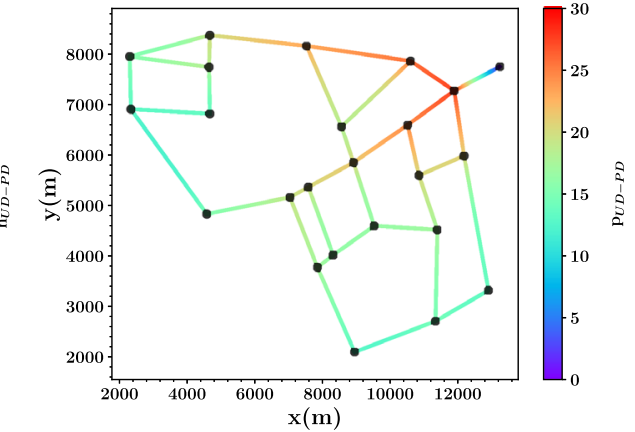

(b)

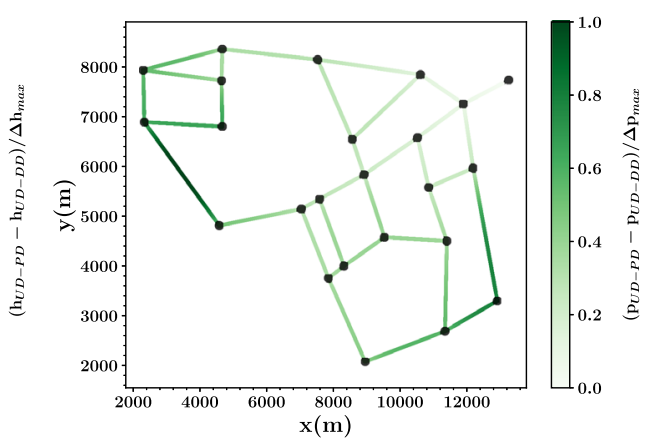

(d)

Fig. 5 a Hydraulic head of the Apulian WDN obtained with the UD-PD scheme; b normalized difference between the heads obtained with the UD-DD and UD-PD schemes 
Fig. 4c shows that both distributed models simulate a non linear behaviour in the hydraulic pressure along the pipe $A B$ the pressure differs both along the pipes and in the nodes. On the contrary, the pressure computed in the node $A$ and $B$ with the demand driven models results similar in the nodes and differs from the one computed with the UD-PD. Similar to case $(i)$, both distributed models are able to capture the flow inversion even in case of $D_{\text {tot }, A B}=20$ $l / s$ although the pressure in the UD-PD results higher due the fact less water is withdrawn in case of pressure driven demand. It worth to notice that this results are in agreement with the conclusions presented in Section 4.1 and further underline the differences between the UD-DD and the demand driven models in pressure scarcity conditions.

\subsection{Case Study}

This test case has two purposes: firstly, to show the applicability of the new UD-PD scheme to a real network; and secondly, to demonstrate that the hydraulic head distribution could considerable change when the distributed pressure driven demand is considered. As a consequence, the new UD-PD method has been applied to a real WDN, known in scientific literature as Apulian Network test case (Giustolisi et al. 2008) (see layout and detailed report of the network in Fig. 9 and Table 2 of Electronic Supplementary Material). It has to be underlined that the original nodal water demand presented in Giustolisi et al. (2008)'s work are herein distributed along each pipe to enforce the differences between pressure and demand driven simulations.

Figure 5a and $\mathrm{b}$ illustrate respectively the hydraulic head and the pressure along the Apulian network computed with the UD-PD scheme. As it can be noticed, the higher hydraulic head values are located near the network tank and they decrease as the the distance from the tank increases. As expected, the hydraulic pressure results instead very low near the

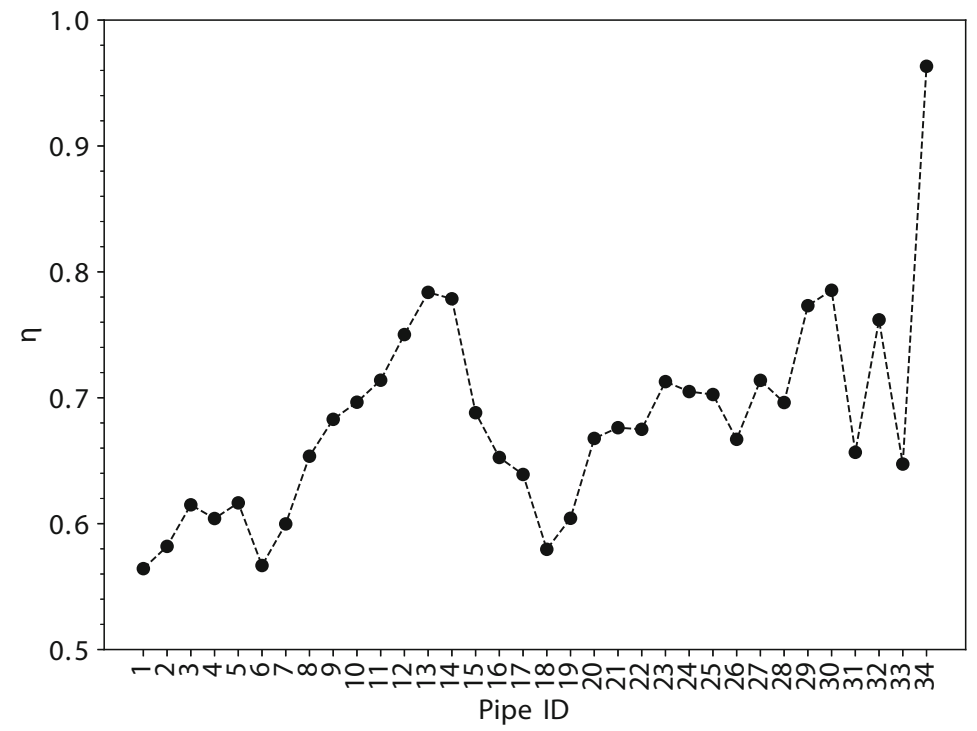

Fig. 6 Normalized difference between pipe water demand and pipe withdrawan water for each pipe 


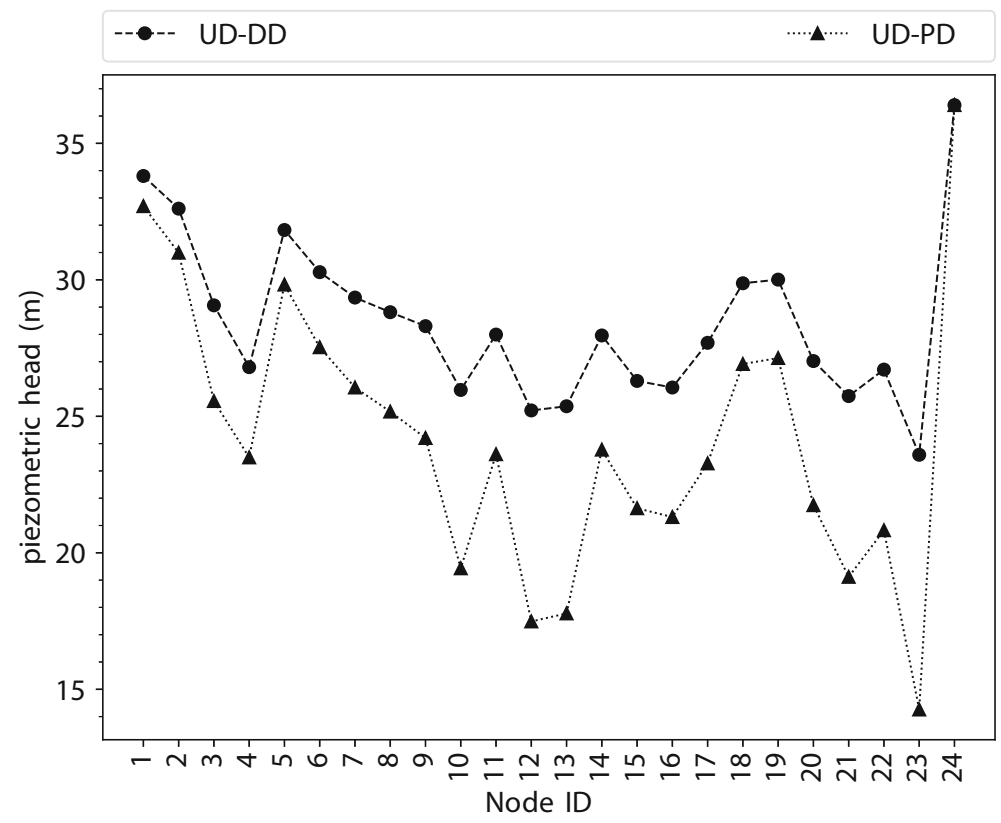

(a)

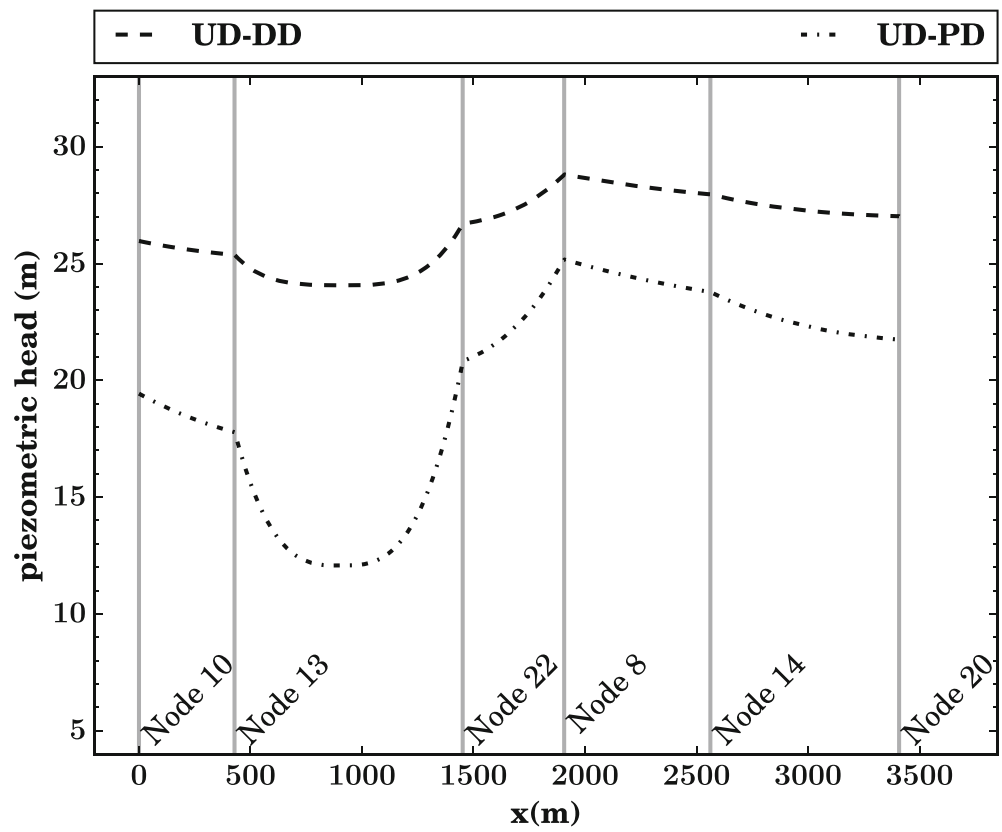

(b)

Fig. 7 Comparison between different scheme for pressure (a) and flow (b) in a reference test case 
tank increases in the nodes adjacent to the tank and reaches again low values in the external pipes of the network. This follows that, according to the pressure-flow relationship used in these these test cases, there is not sufficient pressure to satisfy the water demands along the network pipes. In particular, defying the following parameter:

$$
\eta=\frac{D_{i j, t o t}-W_{i j, t o t}}{D_{i j, t o t}} ;
$$

it is possible to visualize in Fig. 6 the rate of the actual withdrawan water with respect to the real demand water and to quantify the impact of the pressure driven simulation. It worth noticing that according to the definition in Eq. $26 \eta \in[0,1]$, where $\eta=0$ in case that all the water demand, in the selected pipe, is satisfied and $\eta=1$ when no water is delivered. Hence, in pipe 34 , which is the pipe with the lowest pressure, $\eta$ assumes a value very closed to 1 , meaning that almost all the demand is not satisfied. Correspondingly, in the pipes 13 and $14 \eta$ has a value close to 0.8 which means that only the $20 \%$ of the demand water is withdrawn. As reported in Fig. 5c and a, pipes 13 and 14 are not only the pipes where the both the pressures and hydraulic heads are lower but also where the differences between the UD-DD and the UD-PD models are higher. On the contrary, in pipes 1, 6 and 18, where both the hydraulic head and pressure are high, $\eta$ decreases to a value of $0.56,0.56$. and 0.57 which denotes that almost the $40 \%$ of the demand is satisfied. In order to better underline the differences between the UD-DD and UD-PD schemes Fig. 7a shows the hydraulic head in the network noted computed with the two methods and Fig. 7b illustrates the hydraulic heads along some external pipes of the network, which further illustrates that the UDPD even in a real WDN is able to capture both the non linear behaviour of the hydraulic head along the network and to correctly compute the flow inversion in pressure driven conditions.

\section{Conclusion}

This paper presents a new formulation of the GGA scheme in order to properly model uniformly pressure driven demand along pipes in a WDN. In particular, a new approximation of the head loss equation, based on solving high order polynomial equation, is formulated as well an additional step in the iterative Newton-Rapson scheme is introduced. In addition, the new uniformly pressure driven scheme is tested with reference solution to prove its performance and accuracy. Furthermore applications to two WDNs illustrate some comparisons between the uniformly pressure driven scheme and the standard uniformly demand driven scheme. These simulations underline that these two schemes, pressure and demand driven, lead to the same results in case of sufficient pressure in a WDN. On the contrary, the uniformly pressure driven scheme results into a higher nodal pressure and simultaneously a lower withdrawn water where the pressure is not sufficient to deliver all the demand water.

The presented results make the new distributed pressure driven demand head-loss equation formulation within the GGA scheme an essential tool to properly simulate flow and pressure in a WDN in pressure deficit conditions. 
Acknowledgements This work has been partially carried out within the Research project AI-ALPEN, CUP:B26J16000300003 funded by the PAB (Autonomy Provence of Bozen-Bolzano,Italy) for University Research-2014. The EFRE-FESR project Thermo Fluid Dynamics, infrastructures for applied research (ERDF 2014- 2020, CUP: I52F16000850005) support is also acknowledged. The authors thank Professor Righetti Maurizio for his constant guidance and the encouragement in water researches.

\section{Compliance with Ethical Standards}

\section{Conflict of interests None}

Open Access This article is distributed under the terms of the Creative Commons Attribution 4.0 International License (http://creativecommons.org/licenses/by/4.0/), which permits unrestricted use, distribution, and reproduction in any medium, provided you give appropriate credit to the original author(s) and the source, provide a link to the Creative Commons license, and indicate if changes were made.

\section{Appendix A}

The unknown $w_{i j, l}$ coefficients of the approximated withdrawal are the analytical solution of the following linear System:

$$
\left[\begin{array}{cccc}
x_{1}^{n d-1} & \cdots & \cdots & 1 \\
\vdots & \ddots & \vdots & 1 \\
\vdots & x_{l}^{n d-1} & \vdots & 1 \\
\vdots & \ddots & \vdots & 1 \\
x_{n d}^{n d-1} & \cdots & \cdots & 1
\end{array}\right]\left[\begin{array}{c}
\widetilde{w}_{i j, i} \\
\vdots \\
\widetilde{w}_{i j, l} \\
\vdots \\
\widetilde{w}_{i j, n d}
\end{array}\right]=\left[\begin{array}{c}
w_{i j}\left(h_{i j}\left(x_{1}\right)\right) \\
\vdots \\
w_{i j}\left(h_{i j}\left(x_{k}\right)\right) \\
\vdots \\
\left.\left.w_{i j}\left(h_{i j}\right) x_{n d}\right)\right)
\end{array}\right] ;
$$

where, according to pipe $i j$-th notations, $x_{1}=0$ and $x_{k}=L_{i j}$. Specifically, considering a second order approximation, the pressure driven water withdrawal function in the $i j-t h$ pipe reads as:

$$
\widetilde{w}_{i j}(x)=\widetilde{w}_{i j, 1} x^{2}+\widetilde{w}_{i j, 2} x+\widetilde{w}_{i j, 3} ;
$$

which reduces the linear System (27) to:

$$
\left[\begin{array}{lll}
x_{1}^{2} & x_{1} & 1 \\
x_{2}^{2} & x_{2} & 1 \\
x_{3}^{2} & x_{3} & 1
\end{array}\right]\left[\begin{array}{l}
\tilde{w}_{i j, 1} \\
\tilde{w}_{i j, 2} \\
\tilde{w}_{i j, 3}
\end{array}\right]=\left[\begin{array}{l}
w_{i j}\left(h_{i j}\left(x_{1}\right)\right) \\
w_{i j}\left(h_{i j}\left(x_{2}\right)\right) \\
w_{i j}\left(h_{i j}\left(x_{3}\right)\right)
\end{array}\right] ;
$$

and where $\left(x_{1}, x_{2}, x_{3}\right)^{T}=\left(0, L_{i j} / 2, L_{i j} / 2\right)^{T}$. The final coefficients are therefore:

$$
\begin{aligned}
& \widetilde{w}_{i j, 1}=\frac{w_{i j}\left(h_{i j}(0)\right)+w_{i j}\left(h_{i j}\left(L_{i j}\right)\right)-2 w_{i j}\left(h_{i j}\left(\frac{L_{i j}}{2}\right)\right)}{L_{i j}^{2}} ; \\
& \widetilde{w}_{i j, 2}=\frac{3 w_{i j}\left(h_{i j}(0)\right)+w_{i j}\left(h_{i j}\left(L_{i j}\right)\right)-4 w_{i j}\left(h_{i j}\left(\frac{L_{i j}}{2}\right)\right)}{L_{i j}} ; \\
& \widetilde{w}_{i j, 3}=w_{i j}\left(h_{i j}\left(L_{i j}\right)\right) .
\end{aligned}
$$




\section{Appendix B}

Considering second order approximation, the pressure driven water withdrawal function in the $i j-t h$ pipe reads as:

$$
\widetilde{w}_{i j}(x)=\widetilde{w}_{i j, 1} x^{2}+\widetilde{w}_{i j, 2} x+\widetilde{w}_{i j, 3}
$$

hence the water flow in $i j-t h$ pipe results as:

$$
Q_{i j}(x)=Q_{i j}-\left(\frac{1}{3} \widetilde{w}_{i j, 1} x^{3}+\frac{1}{2} \widetilde{w}_{i j, 2} x^{2}+\widetilde{w}_{i j, 3} x\right) ;
$$

According to the Darcy-Weisbach and under the hypothesis of fully turbolente hydraulic regime, the hydraulic head loss between the $i$ - $t h$ and the $j$-th node connected by the $i j$-th reads as:

$$
\begin{aligned}
\Delta h_{i j}= & \int_{0}^{L_{i j}} r_{i j} Q_{i j}(x)^{2} d x ; \\
= & \int_{0}^{L_{i j}} r_{i j}\left(Q_{i j}-\frac{1}{3} \widetilde{w}_{i j, 1} x^{3}-\frac{1}{2} \widetilde{w}_{i j, 2} x^{2}-\widetilde{w}_{i j, 3} x\right)^{2} d x ; \\
= & \frac{r_{i j} \widetilde{w}_{i j, 1}^{2} L_{i j}^{7}}{63}+\frac{r_{i j} \widetilde{w}_{i j, 1} \widetilde{w}_{i j, 2} L_{i j}^{6}}{18}+\frac{r_{i j} L_{i j}^{5}}{5}\left(\frac{\widetilde{w}_{i j, 3} \widetilde{w}_{i j, 2}}{3}+\frac{\widetilde{w}_{k, 2}^{2}}{4}\right) \\
& +\frac{r_{i j} L_{i j}^{4}}{4}\left(-\frac{2 Q_{i j} \widetilde{w}_{i j, 1}}{3}+\widetilde{w}_{i j, 2} \widetilde{w}_{i j, 3}\right)+\frac{r_{i j} L_{i j}^{3}}{3}\left(-2 Q_{i j} \widetilde{w}_{i j, 2}+\widetilde{w}_{i j, 3}^{2}\right) \\
& -r_{i j} \widetilde{w}_{i j, 3} Q_{i j} L_{i j}^{2}-r_{i j} Q_{i j}^{2} L_{i j} ;
\end{aligned}
$$

Moreover, computing the water flow in the $j$-th node of the $i j$-th pipe according to Eq. 34 as:

$$
Q_{j i}=Q_{i j}-\left(\frac{1}{3} \widetilde{w}_{i j, 1} L_{i j}^{3}+\frac{1}{2} \widetilde{w}_{i j, 2} L_{i j}^{2}+\widetilde{w}_{i j, 3} L_{i j}\right)
$$

the amount of the total withdrawal $W_{i j, t o t}$ in the $i j$-th is:

$$
W_{i j, t o t}=\left(\frac{1}{3} \widetilde{w}_{i j, 1} L_{i j}^{3}+\frac{1}{2} \widetilde{w}_{i j, 2} L_{i j}^{2}+\widetilde{w}_{i j, 3} L_{i j}\right) .
$$

At this point, in order to simplify the annotation, we introduce the following definition:

$$
W_{i j, d}=\left(\frac{1}{3} \widetilde{w}_{i j, 1} L_{i j}^{2}+\frac{1}{2} \widetilde{w}_{i j, 2} L_{i j}+\widetilde{w}_{i j, 3}\right) .
$$

and re-write $L_{i j}$ as:

$$
L_{i j}=\frac{Q_{i j}-Q_{j i}}{W_{i j, t o t}}
$$


Substituting Eqs. 36, 37 and 39 into 35 and taking into account the sign of the flows $Q_{i j}$ and $Q_{j i}$ is possible to get the general formulation for the head loss in the $i j$-th pipe as:

$$
\begin{aligned}
\Delta h_{i j} & =\frac{r_{i j}\left(\left|Q_{i j}\right|-\left|W_{i j, t o t}-Q_{i j}\right|\right)^{5}}{5 W_{i j, d}}\left(\frac{2 \widetilde{w}_{i j, 3} \widetilde{w}_{i j, 1}}{3}+\frac{\widetilde{w}_{i j, 2}^{2}}{4}\right)+ \\
& +\frac{r_{i j}\left(\left|Q_{i j}\right|-\left|W_{i j, t o t}-Q_{i j}\right|\right)^{4}}{4 W_{i j, d}^{4}}\left(\widetilde{w}_{i j, 3} \widetilde{w}_{i j, 2}-\frac{2 Q_{i j} \widetilde{w}_{i j, 1}}{3}\right) \frac{\left|Q_{i j}\right|-\left|W_{i j, t o t}-Q_{i j}\right|}{|| Q_{i j}|-| W_{i j, t o t}-Q_{i j}||} \\
& +\frac{r_{i j}\left(\left|Q_{i j}\right|-\left|W_{i j, t o t}-Q_{i j}\right|\right)^{4}\left(\widetilde{w}_{i j, 3}^{2}-Q_{i j} \widetilde{w}_{i j, 2}\right)}{3 W_{i j, d}^{3}}+ \\
& +\frac{r_{i j}\left(\left|W_{i j, t o t}-Q_{i j}\right|-\left|Q_{i j}\right|\right)^{6}\left(\widetilde{w}_{i j, 1} \widetilde{w}_{i j, 2}\right)}{18 W_{i j, d}^{6}} \frac{\left|Q_{i j}\right|-\left|W_{i j, t o t}-Q_{i j}\right|}{|| Q_{i j}|-| W_{i j, t o t}-Q_{i j}||}+ \\
& -\frac{r_{i j}\left(\left|W_{i j, t o t}-Q_{i j}\right|-\left|Q_{i j}\right|\right)^{7} \widetilde{w}_{i j, 1}^{2}}{63 W_{i j, d}^{7}}+ \\
& -\frac{r_{i j}\left|Q_{i j}\right|\left(\left|W_{i j, t o t}-Q_{i j}\right|-\left|Q_{i j}\right|\right)^{2} \widetilde{w}_{i j, 3}}{W_{i j, d}^{2}}+\frac{r_{i j} Q_{i j}^{2}\left(\left|Q_{i j}\right|-\left|W_{i j, t o t}-Q_{i j}\right|\right)}{W_{i j, d}} .
\end{aligned}
$$

According to the Taylor series discussed in Section 3, is it possible to derived the Eq. 40 with respect to the flow $Q_{i j}$, which resulting as:

$$
\begin{aligned}
& \frac{d}{d Q_{i j}} \Delta h_{i j}=\left(\frac{r_{i j}\left(\left|Q_{i j}\right|-\left|W_{i j, t o t}-Q_{i j}\right|\right)^{4}}{W_{i j, d}^{5}}\left(\frac{2 \widetilde{w}_{i j, 3} \widetilde{w}_{i j, 1}}{3}+\frac{\widetilde{w}_{i j, 2}^{2}}{4}\right)\right. \\
& +\frac{r_{i j}\left(\left|Q_{i j}\right|-\left|W_{i j, t o t}-Q_{i j}\right|\right)^{3}}{W_{i j, d}^{5}}\left(\widetilde{w}_{i j, 3} \widetilde{w}_{i j, 2}-\frac{2 Q_{i j} \widetilde{w}_{i j, 1}}{3}\right) \\
& \left.\frac{\left|Q_{i j}\right|-\left|W_{i j, t o t}-Q_{i j}\right|}{|| Q_{i j}|-| W_{i j, t o t}-Q_{i j}||}\right)\left(\frac{Q_{i j}}{\left|Q_{i j}\right|}+\frac{W_{i j, t o t}-Q_{i j}}{\left|W_{i j, t o t}-Q_{i j}\right|}\right) \\
& -\left(\frac{r_{i j}\left(\left|Q_{i j}\right|-\left|W_{i j, t o t}-Q_{i j}\right|\right)^{4} \widetilde{w}_{i j, 1}}{6 W_{i j, t o t}^{4}}\right) \frac{\left|Q_{i j}\right|-\left|W_{i j, t o t}-Q_{i j}\right|}{|| Q_{i j}|-| W_{i j, t o t}-Q_{i j}||} \\
& +\left(\frac{R_{i j}\left(\left|Q_{i j}\right|-\left|W_{i j, t o t}-Q_{i j}\right|\right)^{4} \widetilde{w}_{i j, 1}}{4 W_{i j}^{4}}\right)\left(\widetilde{w}_{i j, 3} \widetilde{w}_{i j, 2}-\frac{2 Q_{i j} \widetilde{w}_{i j, 1}}{3}\right) \\
& \left(\frac{Q_{i j}}{\left|Q_{i j}\right|}-\frac{W_{i j, t o t}-Q_{i j}}{\left|W_{i j, t o t}-Q_{i j}\right|}\right)+ \\
& \frac{R_{i j}\left(\left|Q_{i j}\right|-\left|W_{i j, t o t}-Q_{i j}\right|\right)^{2}\left(\widetilde{w}_{i j, 3}^{2}-Q_{i j} \widetilde{w}_{i j, 2}\right)}{W_{i j, d}^{3}}\left(\frac{\left|Q_{i j}\right|-\left|W_{i j, t o t}-Q_{i j}\right|}{|| Q_{i j}|-| W_{i j, t o t}-Q_{i j}||}\right) \\
& -\frac{r_{i j}\left(\left|Q_{i j}\right|-\left|W_{i j, t o t}-Q_{i j}\right|\right)^{3} \tilde{w}_{i j, 2}}{3 W_{i j, d}}+\frac{r_{i j}\left(-\left|Q_{i j}\right|+\left|W_{i j, t o t}-Q_{i j}\right|\right)^{6} \widetilde{w}_{i j, 1}^{2}}{9 W_{i j, d}^{7}} \\
& \left(\frac{\left|Q_{i j}\right|+\left|W_{i j, t o t}-Q_{i j}\right|}{|| Q_{i j}|+| W_{i j, t o t}-Q_{i j}||}\right)+\frac{r_{i j} Q_{i j}}{\left|Q_{i j}\right| W_{i j, d}^{2}}\left(\left|W_{i j, t o t}-Q_{i j}\right|-\left|Q_{i j}\right|\right)^{2} \widetilde{w}_{i j, 3} \\
& -\frac{2 r_{i j} Q_{i j}}{\left|Q_{i j}\right| W_{i j, d}^{2}}\left(\left|W_{i j, t o t}-Q_{i j}\right|-\left|Q_{i j}\right|\right) \widetilde{w}_{i j, 3}\left(\frac{\left|Q_{i j}\right|+\left|W_{i j, t o t}-Q_{i j}\right|}{|| Q_{i j}|+| W_{i j, t o t}-Q_{i j}||}\right) \text {; }
\end{aligned}
$$

whose inverse is the coefficient $a_{i j}$. 
Publisher's Note Springer Nature remains neutral with regard to jurisdictional claims in published maps and institutional affiliations.

\section{References}

Ang WK, Jowitt PW (2006) Solution for water distribution systems under pressure-deficient conditions. J Water Resour Plan Manag 132(3):175-182. https://doi.org/10.1061/(ASCE)0733-9496(2006)132:3(175)

Avesani D, Righetti M, Righetti D, Bertola P (2012) The extension of EPANET source code to simulate unsteady flow in water distribution networks with variable head tanks. J Hydroinformatics 14(4):960. https://doi.org/10.2166/hydro.2012.013

Berardi L, Giustolisi O, Todini E (2010) Accounting for uniformly distributed pipe demand in wdn analysis: enhanced gga. Urban Water J 7(4):243-255. https://doi.org/10.1080/1573062X.2010.491550

Boulos P, Altman T (1991) A graph-theoretic approach to explicit nonlinear pipe network optimization. App Math Model 15(9):459-466. https://doi.org/https://doi.org/10.1016/0307-904X(91)90035-N. http:// www.sciencedirect.com/science/article/pii/0307904X9190035N

Conte G, Bolognesi A, Bragalli C, Branchini S, Carli AD, Lenzi C, Masi F, Massarutto A, Pollastri M, Principi I (2012) Innovative urban water management as a climate change adaptation strategy: Results from the implementation of the project "water against climate change (wataclic)". Water 4(4):10251038. https://doi.org/10.3390/w4041025. http://www.mdpi.com/2073-4441/4/4/1025

Cross H (1936) Analysis of flow in networks of conduits or conductors. Tech rep., University of Illinois at Urbana Champaign, College of Engineering. Engineering Experiment Station

Dirac PAM (1939) A new notation for quantum mechanics. Math Proc Camb Philos Soc 35(3):416-418. https://doi.org/10.1017/S0305004100021162

Epp R, Fowler A (1970) Efficient code for steady-state flows in network. no. 12

Giustolisi O (2010) Considering actual pipe connections in water distribution network analysis. J Hydraul Eng 136(11):889-900. https://doi.org/10.1061/(ASCE)HY.1943-7900.0000266

Giustolisi O, Todini E (2009) Pipe hydraulic resistance correction in WDN analysis. Urban Water J 6(1):3952. https://doi.org/10.1080/15730620802541623

Giustolisi O, Savic D, Kapelan Z (2008) Pressure-driven demand and leakage simulation for water distribution networks. J Hydraul Eng 134(5):626-635. https://doi.org/10.1061/(ASCE)0733-9429(2008)134: $5(626)$

Ingeduld P (2007) Real-time forecasting with EPANET. https://doi.org/10.1061/40927(243)508

Ingeduld P, Pradhan A, Svitak z, Terrai A (2006) Modelling intermittent water supply systems with EPANET. https://doi.org/10.1061/40941(247)37

Jakob M, Steckel JC (2016) Implications of climate change mitigation for sustainable development. Environ Res Lett 11(10):104010. http://stacks.iop.org/1748-9326/11/i=10/a=104010

Kalungi P, Tanyimboh TT (2003) Redundancy model for water distribution systems. Reliab Eng Syst Saf 82(3):275-286. https://doi.org/10.1016/S0951-8320(03)00168-6. http://www.sciencedirect. com/science/article/pii/S0951832003001686

Menapace A, Avesani D, Righetti M (2018) Uniformly distributed demand EPANET extension. Water Resour Manage. https://doi.org/10.1007/s11269-018-1924-6, Pisaturo G

Murty Y (1972) Use of digital computers for pipe network analysis. University of Wisconsin, Madison. https://books.google.it/books?id=TqRfAAAAMAAJ

Oki T, Kanae S (2006) Global hydrological cycles and world water resources. Science 313(5790):1068-1072. https://doi.org/10.1126/science.1128845. http://science.sciencemag.org/content/313/5790/1068, http:// science.sciencemag.org/content/313/5790/1068.full.pdf

Ormsbee LE (2006) The history of water distribution network analysis: the computer age. In: 8th annual water distribution systems analysis symposium

Press WH, Teukolsky SA, Vetterling WT, Flannery BP (2007) Numerical recipes 3rd edition: the art of scientific computing, 3rd edn. Cambridge University Press, New York

Rossman LA (2000) EPANET 2: Users manual. Cincinnati US Environmental Protection Agency National Risk Management Research Laboratory 38(September):200. https://doi.org/10.1177/0306312708089715

Rossman LA (2007) Discussion of solution for water distribution systems under pressure-deficient conditions; by Wah Khim Ang and Paul W. Jowitt. J Water Resour Plan Manag 133(6):566-567. https://doi.org/10.1061/(ASCE)0733-9496(2007)133:6(566.2)

Siew C, Tanyimboh TT (2012) Pressure-dependent epanet extension. Water Resour Manag 26(6):1477-1498. https://doi.org/10.1007/s11269-011-9968-x. https://doi.org/10.1007/s11269-011-9968-X 
Tanyimboh TT, Templeman AB (2010) Seamless pressure-deficient water distribution system model. Proc Inst Civ Eng Water Manage 163(8):389-396. https://doi.org/10.1680/wama.900013

Todini E, Pilati S (1988) Computer applications in water supply: Vol. 1—systems analysis and simulation. In: A gradient algorithm for the analysis of pipe networks. Research Studies Press Ltd., Taunton, pp 1-20. http://dl.acm.org/citation.cfm?id=61052.61053

Wu ZY, Clark C (2009) Evolving effective hydraulic model for municipal water systems. Water Resour Manag 23(1):117-136. https://doi.org/10.1007/s11269-008-9267-3 